\title{
Personalized E-Learning Recommender System using Multimedia Data
}

\author{
Hayder Murad \\ School of Engineering \\ University of Portsmouth \\ Portsmouth, United Kingdom
}

\author{
Linda Yang \\ School of Engineering \\ University of Portsmouth \\ Portsmouth, United Kingdom
}

\begin{abstract}
Due to the huge amounts of online learning materials, e-learning environments are becoming very popular as means of delivering lectures. One of the most common e-learning challenges is how to recommend quality learning materials to the students. Personalized e-learning recommender systems help to reduce information overload, which tailor learning material to meet individual student's learning needs. This research focuses on using various recommendation and data mining techniques for personalized learning in e-learning environment.
\end{abstract}

Keywords-E-Learning; recommender system; data mining

\section{PURPOSE}

The explosive growth in online learning materials has generated an urgent need for new techniques and access mechanisms to reduce information overload. eLearning recommender system has been paid a great attention by the researchers in the field of recommender system to solve the problem of information overload in learning materials and personalisation [1]-[3]. This research aims to develop a personalized e-learning recommender system to undergraduate students based on students' level of knowledge, and students' profiles with the use of online video learning materials.

The objectives of the project include:

- Developing a framework for supporting the development of the personalized eLearning recommender system.

- Developing a classification method to classify online video learning materials.

- Developing a profiling method to extract student profile and level of knowledge.

- A prototype will be designed as an intelligent tutor to save the time and effort of the lecturer and also increasing self-learning skills.

In this paper, we report on the design of the framework, which is able to detect students' profiles and their level of knowledge and automatically recommend online video learning material that meets the student needs.

\section{BACKGROUND/SIGNIFICANCE}

UK universities accept thousands of international students every year, some of those students get accepted directly into second or third year through agreements between institutions which called 'Direct Entry programme'.
Direct Entry programme accepts student according to specific requirements like qualifications and grade certificates without taking into account the learning styles in their countries, differences between learning materials in UK and their countries and knowledge level of the students in the courses which they applied to. These may cause a problem to the student during his study in the University, also the instructor will suffer from this situation as he/she will teach students have lack of information about the courses which they teach. From this problem the proposed idea tries to solve such problems by offering a personalised e-learning recommender system to help direct entry student to reduce the lack of information by estimating their knowledge level and after that online video learning materials will be recommended to them.

The goal of a Recommender System is to generate meaningful recommendations to a collection of users for items or products that might interest them. Suggestions for books on Amazon, or movies on Netflix, are real world examples of the operation of industry strength Recommender systems [4]. In other words, Recommender systems are information agents that attempt to predict which items out of large pool a user may be interested in and Recommend the best one to the target user [5].

Due to the huge amounts of online learning materials, eLearning environments are becoming very popular as means of delivering lectures. The challenge of eLearning environments with the existence of large number of learning materials is how to recommend quality learning materials to the students when they have limited time to view and study [6]. Also, due to the large development in internet in term of speed and the wide use of mobile phones and tablets with video capabilities, all these reasons made the use of video in eLearning very popular. Learning via video became one of the popular ways of education and brings new dimensions to the learning methods. For example, if certain course provide contents that include practical skills like building a PC or designing a network using simulator, these part of the course will be most benefit to see it in video rather than explained by text and static images [7].

Accordingly, eLearning recommender system has been paid a great attention by the researchers in the field of recommender system to solve the problem of information overload in learning materials and personalisation [1]-[3].

This project is sponsored by Ministry of Higher Education and Scientific Research in Iraq. 
eLearning recommender system aims to predict suitable learning materials from large amount of learning data based on student preferences [8], [9].

Research contributions organised as following:

- Define a framework to support the development of personalised eLearning recommender system.

- Develop a dynamic hierarchical classification method/algorithm to classify online video learning materials using machine learning and information retrieval techniques.

- Develop a user profiling method to model student's learning need and context determinations.

- Design personalised recommender system algorithm to filter learning materials to meet students' personal needs.

\section{METHOD}

\section{A. Design of the Proposed Framework}

As shown in Fig. 1, the proposed framework consists of two sides: the client side and the server side.

The server side:

- Student profiling module: processes student context information whether it's explicit or implicit.

- Knowledge estimation module: gathers data to produce suitable information about a student's knowledge level.

- Feedback module: deals with feedback retrieved from students to refine the recommendation process.

- Assessment module: provides dynamic assessment.

- Recommendation module: recommends learning material to individual student based on student's knowledge and profile.

- Video searcher and classifier: classify and categorize video learning materials.

The client side:

- User interface module: it's responsible for all transactions that can be happened between the web and computer.

- Student profile: it's responsible for observe student usage behaviour and store it in student profiling module.

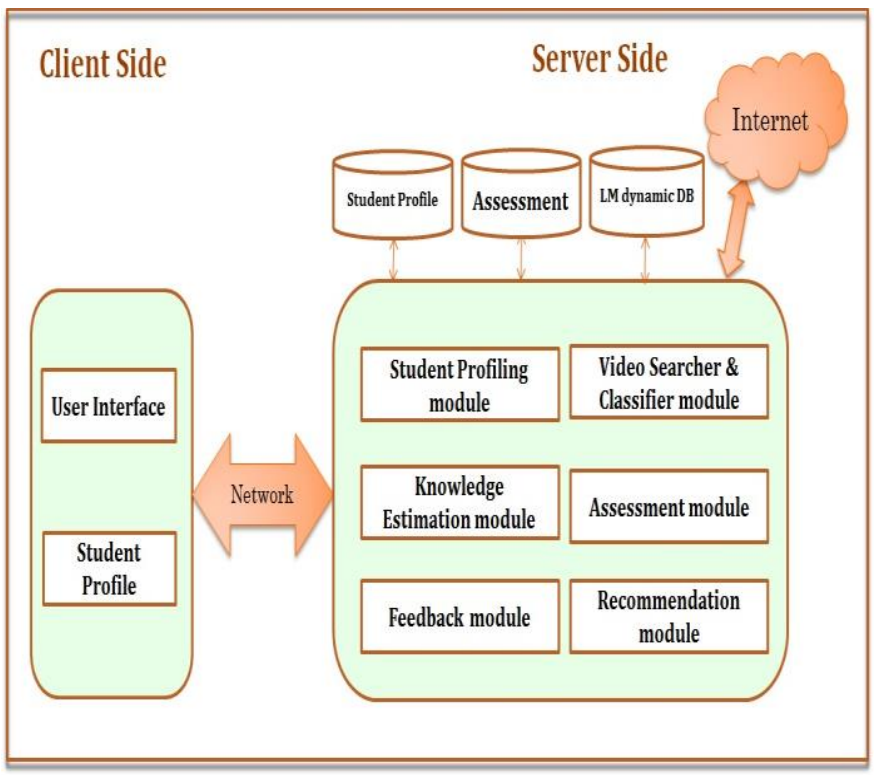

Fig. 1. Proposed framework.

\section{B. Data Collection}

The required online learning materials will be retrieved from online video websites (initially YouTube) and classified and saved its links in dynamic learning materials repository to be recommended to the student.

The metadata of YouTube online videos are extracted using video searcher and classifier module. This metadata includes uploader dependent metadata (titles and descriptions) and uploader independent metadata (comments, No. of views, likes, dislikes ... etc.) as shown in Fig. 2.

The proposed methodology to extract the metadata from YouTube videos and classify them based on the extracted metadata by applying text mining techniques into topics and subtopics are shown in Fig. 3.

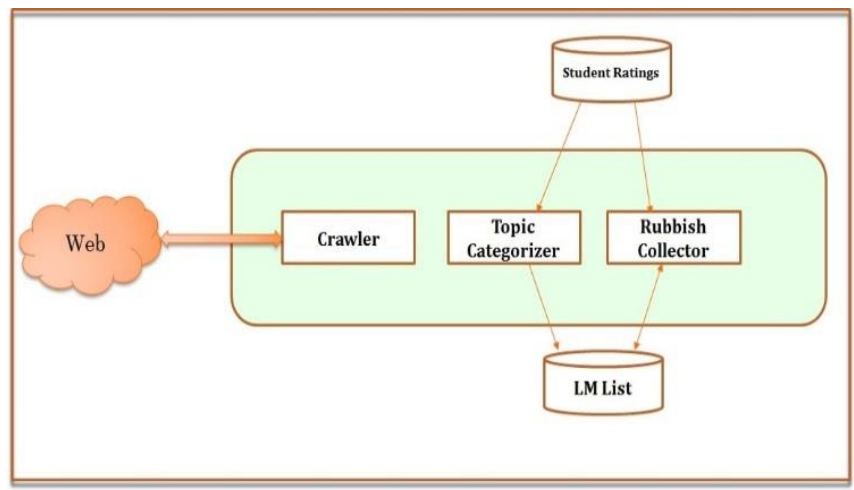

Fig. 2. Video searcher and classifier. 


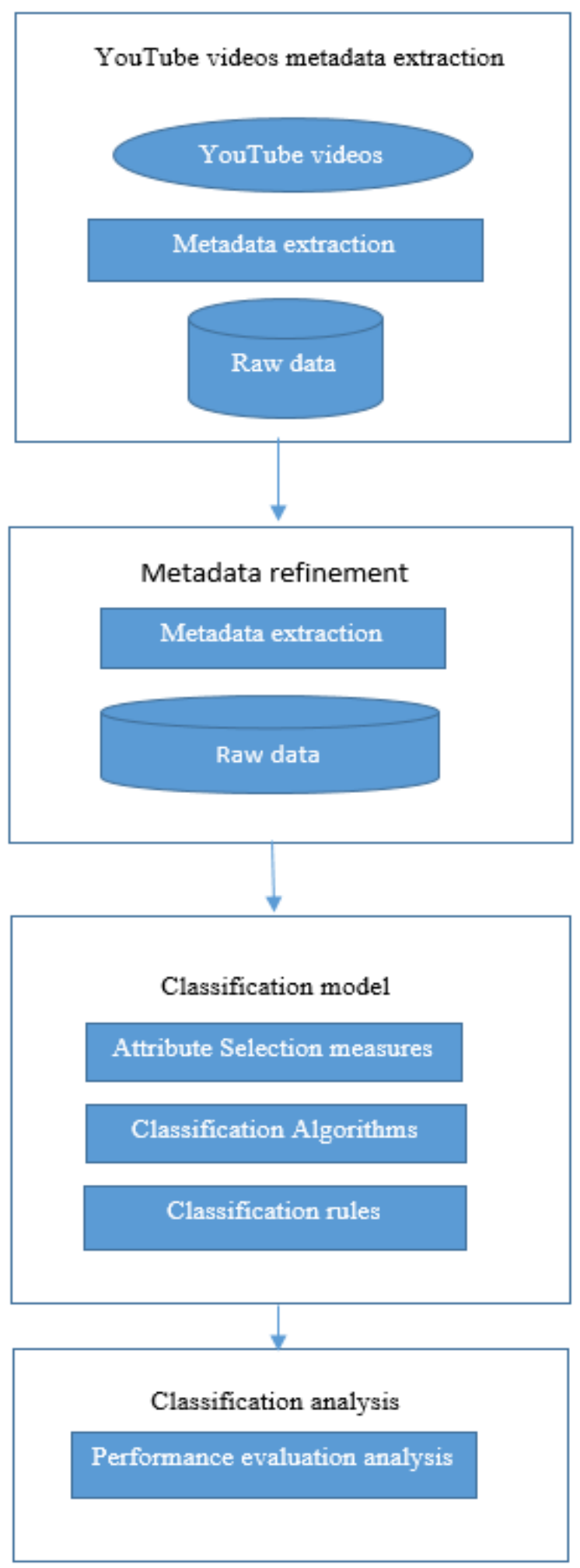

Fig. 3. Methodology.

\section{CONCLUSION AND FUTURE WORK}

This proposed framework supports the development of personalised eLearning recommender system combining the techniques of student profiling, knowledge estimation, assessment and feedbacks in order to improve students learning process by recommending online video learning materials based on student profile and knowledge level. This paper explains the work process which is going to be implemented using algorithms that will be designed to support the system. Previous researches have been focused on text - based learning material. What we are doing is to build the foundation for processing and mining of online multimedia data, and put this work into engineering practices. Based on the proposed framework an initial prototype and a classification method have been developed. Our work is just at the beginning and far from accomplishment. In the near future, we would like to improve a dynamic Hierarchical classification method to classify online video learning material, as well as develop a user profiling method to model student's learning need and context determinations. We believe that personalised recommender is necessary for tailoring learning materials to meet individual student's learning need. We will try to build such a personalised recommender system algorithms to filter learning materials to meet students' personal needs.

\section{ACKNOWLEDGMENT}

This project is funded by Ministry of Higher Education and Scientific Research in Iraq (MOHE) and the represented of the ministry is the Iraqi Cultural Attach in London.

\section{REFERENCES}

[1] C. Chen, L. Duh, and C. Liu, "A personalized courseware recommendation system based on fuzzy item response theory," IEEE Int. Conf. e-Technology, e-Commerce e-Service, 2004. EEE '04. 2004, pp. 305-308, 2004.

[2] Y. Gu, "Research on Personalized Recommendation System in ELearning Based on Semantic Web," pp. 1046-1053, 2013.

[3] M. Holenko Dlab and N. Hoic-Bozic, "Recommender system for Web 2.0 supported eLearning," IEEE Glob. Eng. Educ. Conf. EDUCON, no. April, pp. 953-956, 2014.

[4] P. M. V.Sindhwani, "Recommender systems," Commun. ACM, pp. 121, 2010.

[5] S. Akshita, "Recommender System: Review", vol. 71, no. 24, pp. 38-42, 2013.

[6] K. I. Ghauth and N. A. Abdullah, "The effect of incorporating good learners' ratings in e-learning contentbased recommender system," Educ. Technol. Soc., vol. 14, no. 2, pp. 248-257, 2011.

[7] Epignosis LLC, “e-learning Concepts, Trends, Applications," Book, p. $5,2014$.

[8] N. Soonthornphisaj, E. Rojsattarat, and S. Yim-ngam, "Computational Intelligence: International Conference on Intelligent Computing, ICIC 2006 Kunming, China, August 16-19, 2006 Proceedings, Part II,” D.-S. Huang, K. Li, and G. W. Irwin, Eds. Berlin, Heidelberg: Springer Berlin Heidelberg, 2006, pp. 518-523.

[9] K. Verbert, N. Manouselis, X. Ochoa, M. Wolpers, H. Drachsler, I. Bosnic, and E. Duval, " Context-aware recommender systems for learning: A survey and future challenges," IEEE Trans. Learn. Vol.5, no.4, pp.318-335,2012. 